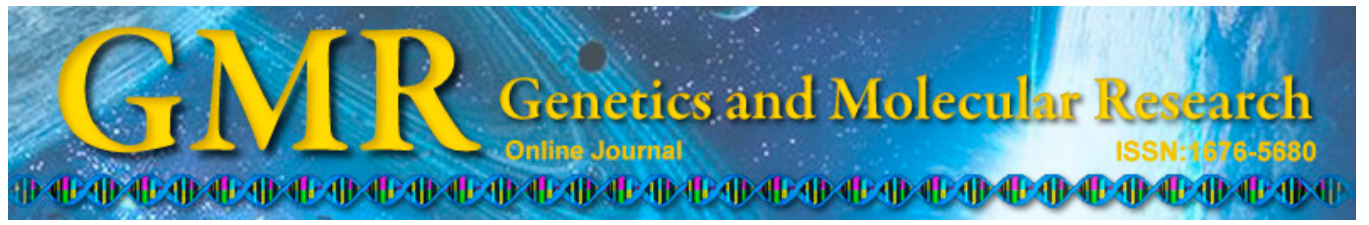

\title{
Role of autoantibodies to various Ro60 epitopes in the decrease of lymphocytes seen in systemic lupus erythematosus and primary Sjögren's syndrome
}

\author{
Z.W. Shuai, Y. Huang, L. Zhang, J. Cai and M. Li \\ Department of Rheumatology and Immunology, \\ The First Affiliated Hospital of Anhui Medical University, Hefei, China \\ Corresponding author: Z.W. Shuai \\ E-mail: shuaizongwen@medmail.com.cn
}

Genet. Mol. Res. 14 (3): 10096-10102 (2015)

Received December 4, 2014

Accepted May 11, 2015

Published August 21, 2015

DOI http://dx.doi.org/10.4238/2015.August.21.17

\begin{abstract}
We investigated the roles of autoantibodies to different Ro60 epitopes in lymphopenia in systemic lupus erythematosus (SLE) and primary Sjögren's syndrome (pSS). We recruited 16 patients with SLE, 14 with pSS, and 10 healthy controls; all were female. Patients had active disease, had not received glucocorticoid or immunosuppressants for at least 3 months, and had positive laboratory tests for autoantibodies against Ro60. Patient peripheral blood lymphocyte (LC) counts were $<1$ x $10^{9} / \mathrm{L}:(0.66 \pm 0.12) \times 10^{9} / \mathrm{L}$ and $(0.70 \pm 0.16) \times 10^{9} / \mathrm{L}$ for SLE and $\mathrm{pSS}$ groups, respectively $(\mathrm{P}=0.511)$. LCs from each group were cultured in vitro with each of the three immunotoxins (ITs) (AE1-3), which specifically combine with one of the three epitopes (aa482-493, aa310323 , and aa230-241, respectively) on Ro60. The cytotoxicity of each IT to the cultured LCs was measured by the MTT colorimetric method. The relationships between IT cytotoxicity and LC counts were analyzed, and autoantibodies against the three epitopes in patient peripheral blood were detected. All ITs showed cytotoxicity to control LCs; however, $\mathrm{AE} 3$ and AE2 showed greater toxicity to LCs from SLE and pSS groups,
\end{abstract}


respectively, and the enhanced cytotoxicity was significantly associated with the respective LC counts $(r=0.653, \mathrm{P}=0.06 ; r=0.594, \mathrm{P}=0.025)$. No difference was found in the prevalence of the autoantibodies between the SLE and pSS groups. These results suggest that autoantibodies to Ro60 might play a pathogenic role in lymphopenia in both SLE and pSS, but the pathogenic mechanisms might differ.

Key words: Lupus erythematosus systemic; Immunotoxin; Primary Sjögren's syndrome; Lymphopenia; Ro60 autoantibody

\section{INTRODUCTION}

Antibodies against SSA/Ro have been found in connective tissue diseases such as Systemic lupus erythematosus (SLE), primary Sjögren's syndrome (pSS), and rheumatoid arthritis. The positive antibody rate to Ro in SLE and SS was reported to be $40-90$ and $70-100 \%$, which is in accordance with the various damages associated with these disorders (Yoshimi et al., 2012). Its antigens included 60- and 52-kDa nucleoproteins. The ribonucleoprotein was composed of 60-kDa SSA/Ro (Ro60) and YRNA, which is present throughout the nucleus and cytoplasm of human tissue cells. Decreased lymphocyte cell (LC) counts were shown to be a common clinical finding in SLE and pSS, although the mechanism remained unclear. Ro60 has 20 epitopes, and synthetic epitopes showed similar antigenicity as the original (Huang et al., 1995; Scofield et al., 1997). The autoantibodies against different epitopes might be associated with various types of clinical damage (Li et al., 2003). In this study, the monoclonal immunotoxins (ITs) of three different epitopes of Ro60 were reacted with peripheral blood LCs from patients with SLE and pSS cultured in vitro, to investigate the possible role of the various epitopes of Ro60 autoantibodies in the decreased LC count in patients compared with healthy controls.

\section{MATERIAL AND METHODS}

\section{Subjects}

We selected 30 patients to participate in this study from the Rheumatology Department of the First Affiliated Hospital of Anhui Medical University from May 2012 to May 2013; all subjects were women. Subjects included 16 patients with SLE, diagnosed in accordance with the classification standard of SLE (Hochberg, 1997); patients with secondary Sjögren syndrome were excluded. The 14 patients with pSS were diagnosed in accordance with the classification standard of pSS (Vitali et al., 2002). Patients in the SLE and pSS groups were aged $36.56 \pm 8.03$ and $34.71 \pm 6.88$ years, $(\mathrm{P}=0.261)$, and the disease courses were $10.63 \pm 5.39$ and $14.00 \pm 5.75$ months $(\mathrm{P}=0.108)$, respectively. The disease condition of all patients was at an active stage, and none had received glucocorticoid and immunosuppressive agent treatments. Antibodies against Ro60 were detected in all patients, and the absolute counts of peripheral blood LCs were all less than $1 \times 10^{9} / \mathrm{L}$; the absolute counts in the SLE and pSS groups were $(0.66 \pm 0.12) \times 10^{9} / \mathrm{L}$ and $(0.70 \pm 0.16) \times 10^{9} / \mathrm{L}$, respectively $(\mathrm{P}=0.511)$. Peripheral blood and clinical materials were collected from each subject. In addition, 10 healthy women aged $35.80 \pm 8.16$ years were selected as the control group. There was no difference in gender and age compared with two groups. This research program was approved by the clini- 
cal research Ethics Committee of the First Affiliated Hospital of Anhui Medical University. The written informed consent was obtained from all participants involved in this study.

\section{Separation and cryopreservation of peripheral blood LCs}

From each patient, $10 \mathrm{~mL}$ peripheral blood was collected in an ethylenediaminetetraacetic acid vacutainer, and was diluted with phosphate buffered saline (PBS) in the ratio of 1:1 at room temperature. The middle layer was collected by centrifugation (1500 rpm, $30 \mathrm{~min}$ ) and was washed in PBS; the supernatant was discarded. RPMI 1640 medium was added to suspend the cells, and the supernatants from 2 subsequent centrifugations were discarded. The final pellet was suspended in RPMI-1640 liquid culture medium containing 5\% dimethyl sulfoxide (DMSO) and $20 \%$ calf serum, and the cell consistency was adjusted to $5-10 \times 10^{7} / \mathrm{mL}$. Cells were cryopreserved using the following protocol: $30 \mathrm{~min}$ at $4^{\circ} \mathrm{C}, 2 \mathrm{~h}$ at $-20^{\circ} \mathrm{C}$, overnight at $-80^{\circ} \mathrm{C}$, followed by storage in liquid nitrogen.

\section{Preparation of the different Ro60 epitope ITs}

Peptides of three epitopes of Ro60: aa482-493 (Epitope1, E1), aa310-323 (E2), and aa230-241 (E3) were synthesized by a stepwise solid phase procedure on a phenylacetamidomethyl resin according to standard methods. A control dipeptide, biotin-Leu-Leu-OH, was also prepared and tested. The identity and purity of the peptides was confirmed by high performance liquid chromatography in the Peptide Synthesis Studio of the Academy of Military Sciences PLA, China. The cDNAs of three single-chain fragment V ( $\mathrm{ScFv}$ ) monoclonal antibodies were selected from the SSA/Ro phage display library (Zhou et al., 2003; Li et al., 2010). The cDNAs of three ScFv monoclonal antibodies against the PE40 fragment cDNA of Pseudomonas aeruginosa exotoxin $\mathrm{A}(\mathrm{PE})$ were constructed by a gene recombination technique. We obtained three ScFv-PE40 cDNAs and transformant Origami bacteria (Novagen, USA). ScFv-PE40 protein was expressed in Origami which was then crushed ultrasonically and purified by NTA-Ni affinity column (Novagen, USA). Then the bifunctionality (antigen specific recognition and cytotoxicity) of each recombinant protein was confirmed (Shuai et al., 2008). The Origami bacteria with the $\mathrm{ScFv}-\mathrm{PE} 40 \mathrm{cDNAs}$ were cryopreserved at $-80^{\circ} \mathrm{C}$, and the $\mathrm{ScFv}-\mathrm{PE} 40$ proteins (AE1, $\mathrm{AE} 2$, and AE3) were obtained prior to the cell culture experiments. Quantification of each protein, which had been sterilized by filter sterilization, was performed using the Bradford method (Bio-Rad protein quantification kit, Bio-Rad Laboratories, Berkeley, CA, USA), and then the proteins were cryopreserved at $-80^{\circ} \mathrm{C}$.

\section{Detection of IT toxicity on LCs by the MTT method}

Cryopreserved LCs were recovered at $40^{\circ} \mathrm{C}$, and 10X volume of RPMI1640 medium was added to suspend the cells; the supernatant was discarded after each of 2 centrifugations (2000 rpm, $6 \mathrm{~min})$. Cells were suspended in fresh RPMI1640 liquid culture medium containing $5 \%$ phytohaemagglutinin, $10 \%$ calf serum, $100 \mu \mathrm{g} / \mathrm{mL}$ streptomycin, and $100 \mu \mathrm{g} /$ $\mathrm{mL}$ penicillin, and cells were maintained at $37^{\circ} \mathrm{C}$ for $72 \mathrm{~h}$ in a humidified atmosphere of $5 \%$ $\mathrm{CO}_{2} / 95 \%$ air. LCs were collected, counted, and then detected by trypan blue staining. Cells were adjusted to a consistency of approximately $1 \times 10^{7} / \mathrm{mL}$; they then were plated on 24-well plates at $100 \mu \mathrm{L} /$ well $\left(1 \times 10^{6}\right.$ cells), with 8 wells for each patient, and medium was added to 1 $\mathrm{mL}$. Cells were maintained for $24 \mathrm{~h}$, and then each of AE1, AE2, AE3, or albumin (ALB) $2 \mu \mathrm{g}$ 
was added in 2 of the eight wells of each patient for another $24 \mathrm{~h}$. Then $50 \mu \mathrm{L} 5 \mathrm{mg} / \mathrm{mL}$ MTT was added in each well for $4 \mathrm{~h}$, and the total culture medium of each well was collected. The supernatant was discarded after centrifugation at $2000 \mathrm{rpm}$ for $10 \mathrm{~min}$. Cells were suspended with $200 \mu \mathrm{L}$ DMSO, and $200 \mu \mathrm{L}$ PBS was added once the cells were completely dissolved. From this solution, $200 \mu \mathrm{L}$ were added to 96 -well plates, and the absorbance was measured at $495 \mathrm{~nm}$. The average value was taken as the detection result.

\section{Detection of serum autoantibodies to E1, E2, and E3 by ELISA}

Aliquots $(50 \mu \mathrm{L})$ of E1, E2, or E3 solution $(100 \mu \mathrm{g} / \mathrm{mL})$ were separately added to 96-well plates as coated antigen at $4^{\circ} \mathrm{C}$ overnight. Goat anti-human IgG (Sigma-Aldrich, St. Louis, MO, USA) conjugated with alkaline phosphatase was utilized as a secondary antibody (1:2000), and the method was performed in accordance with standard ELISA protocol. The detection value of the serum indices in 30 healthy controls (A405 means $+2 \mathrm{~s}$ ) was used as the positive critical value.

\section{Statistical analysis}

Statistical analysis was performed using the SPSS version 19.0 software (SPSS, Chicago, IL, USA). Data are reported as means \pm SE. The $t$-test was used to compare two groups. One-way ANOVA was used to compare three groups. Pearson correlation analysis was used to analyze the correlation. The $\chi^{2}$ test was used to analyze the enumeration data. Differences at the level of $\mathrm{P}<0.05$ were considered to be statistically significant.

\section{RESULTS}

\section{Comparison among different epitope ITs}

The results of the toxicities observed for each group patient LCs treated with IT and ALB using the MTT method are shown in Table 1. The Levene test revealed that the variance of the different indices of the same group and the same indices of different groups were homogeneous. It was suitable to use a one-way ANOVA. The results of different indices of the same group and the same indices of different groups are shown in Table 1. Compared with ALB, the data revealed that the three ITs had some toxicity to the LCs of healthy controls. AE2 and AE3 showed significant toxicity to the LCs of pSS and SLE groups, respectively.

\begin{tabular}{|c|c|c|c|c|c|c|c|}
\hline Group & Patients & AE1 & AE2 & AE3 & ALB & $F$ value ${ }^{\boldsymbol{\Delta}}$ & P value \\
\hline$\overline{\text { SLE }}$ & 16 & $0.432 \pm 0.065$ & $0.424 \pm 0.050$ & $0.351 \pm 0.086^{* \#}$ & $0.536 \pm 0.066^{* \# \#}$ & 20.007 & 0.000 \\
\hline pSS & 14 & $0.441 \pm 0.080$ & $0.340 \pm 0.078^{* \diamond}$ & $0.428 \pm 0.060^{\# \diamond \diamond}$ & $0.527 \pm 0.083^{* \# ※}$ & 14.241 & 0.000 \\
\hline CT & 10 & $0.460 \pm 0.087$ & $0.461 \pm 0.081 \bullet$ & $0.454 \pm 0.084 \diamond$ & 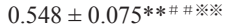 & 2.984 & 0.044 \\
\hline$F$ value $^{\Delta}$ & & 0.440 & 10.149 & 6.476 & 0.234 & & \\
\hline$P$ value ${ }^{\Delta}$ & & 0.647 & 0.000 & 0.004 & 0.793 & & \\
\hline
\end{tabular}

$\mathbf{\Lambda}$ The results of one-way ANOVA in different indices for each group; ${ }^{\wedge}$ the results of one-way ANOVA in the same indices; vs AE1: **0.01 $\leq \mathrm{P}<0.05,{ }^{*} \mathrm{P}<0.01$; vs AE2: ${ }^{*} 0.01 \leq \mathrm{P}<0.05,{ }^{*} \mathrm{P}<0.01 ;$ vs AE3: $* * 0.01 \leq \mathrm{P}<0.05$,

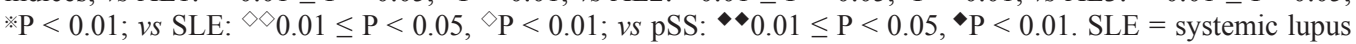
erythematosus; $\mathrm{pSS}=$ primary Sjögren's syndrome; CT = control group; AE1-3 = autoantibody epitopes 1-3; IT = immunotoxin; $\mathrm{ALB}=$ albumin. 


\section{Relationship between LC count and IT toxicity in patients}

The correlations between the peripheral blood LC counts of patients with SLE and the cytotoxicity of different epitope ITs $\left(A_{495}\right.$ value) as well as the correlations between those of patients with pSS were analyzed, and the results are shown in Table 2. AE3 had significant correlation with the LC count in the SLE group, and there was a positive correlation between $\mathrm{AE} 2$ and the LC count in the pSS group.

Table 2. Relationship of LC counts and different IT epitopes in SLE and pSS*.

\begin{tabular}{|c|c|c|c|c|c|c|c|}
\hline \multirow[t]{2}{*}{ Group } & \multirow[t]{2}{*}{ Patients } & \multicolumn{2}{|c|}{ AE1 and LC count } & \multicolumn{2}{|c|}{$\mathrm{AE} 2$ and $\mathrm{LC}$ count } & \multicolumn{2}{|c|}{$\mathrm{AE} 3$ and $\mathrm{LC}$ count } \\
\hline & & $r$ & $P$ & $r$ & $P$ & $r$ & $P$ \\
\hline SLE & 16 & 0.243 & 0.363 & 0.137 & 0.613 & 0.653 & $0.018^{\Delta}$ \\
\hline
\end{tabular}

*The results of Pearson correlation analysis; ${ }^{\wedge} \mathrm{P}<0.05 . \mathrm{LC}=$ lymphocyte; $\mathrm{IT}=$ immunotoxin; $\mathrm{SLE}=$ systemic lupus erythematosus; pSS = primary Sjögren's syndrome; AE1-3 = autoantibody epitopes 1-3.

\section{Detection of autoantibodies to the three epitopes in the peripheral blood of the 2 patient groups}

The results of comparison of the three epitope (AE1, AE2, and AE3) autoantibody positive rates in patients with SLE and pSS are shown in Table 3. There was no significant difference between the two groups in the positive rates of the three epitope autoantibodies.

Table 3. Comparison of the positive rates for autoantibodies for the three epitopes in peripheral blood from patients with SLE and pSS.

\begin{tabular}{|c|c|c|c|c|c|c|c|c|c|c|}
\hline \multirow[t]{2}{*}{ Group } & \multirow[t]{2}{*}{ Patients } & \multicolumn{3}{|c|}{ E1 antibody } & \multicolumn{3}{|c|}{ E2 antibody } & \multicolumn{3}{|c|}{ E3 antibody } \\
\hline & & Positive & Negative & Positive rate & Positive & Negative & Positive rate & Positive & Negative & Positive rate \\
\hline SLE & 16 & 11 & 5 & $68.75 \%$ & 12 & 4 & $75.00 \%$ & 12 & 4 & $75.00 \%$ \\
\hline pSS & 14 & 9 & 5 & $64.29 \%$ & 11 & 3 & $78.57 \%$ & 10 & 4 & $71.43 \%$ \\
\hline & \multicolumn{4}{|c|}{0.067} & \multicolumn{2}{|c|}{0.053} & \multicolumn{4}{|c|}{0.049} \\
\hline $\mathrm{P}$ value & \multicolumn{4}{|c|}{0.796} & \multicolumn{2}{|c|}{0.818} & \multicolumn{4}{|c|}{0.825} \\
\hline
\end{tabular}

$\mathrm{SLE}=$ systemic lupus erythematosus; $\mathrm{pSS}$ = primary Sjögren's syndrome.

\section{DISCUSSION}

LC decrease has been known to be a common feature in patients with SLE and pSS, and it also was a standard of SLE classification diagnosis (Hochberg, 1997). The presence of this feature was important to note, as patients with LC decrease were likely to carry an infection; in addition, LC decrease hindered the clinical application of immunosuppressive therapy. The exact mechanism underlying the LC decrease was unclear, although various autoantibodies had been found in patients with SLE and pSS. One study revealed that the Ro antibody appeared in patients with SLE 3 to 4 months prior to diagnosis (Arbuckle et al., 2003). BALB/c mice immunized with different degrees of oxidative modification of Ro60 (representing different epitopes modified) induced animals different spectrum of damages 
similar to that seen in patients with SLE and SS, which revealed that Ro60 autoantibodies might play an important role in the pathogenesis of the diseases and in the clinical damage seen in SLE and pSS (Kurien et al., 2011). In addition, clinical research has shown that the LC decrease observed in patients with pSS was related to the presence of Ro autoantibodies (Yoshimi et al., 2012). To investigate the possible role of autoantibodies to the various epitopes of Ro60 in the LC decrease in these different diseases, we selected Ro60 antibodies positive patients with SLE and pSS who exhibited LC decrease as subjects. We utilized an in vitro study to avoid the influence of other factors (including other autoantibodies) on the measurement indices that might occur in vivo.

ITs, also known as bispecific antibodies, have bifunctionality: i.e., antigen specific recognition and cytotoxicity, that was not only applicable to studies of targeted cancer therapy (Huang et al., 2012; Zhu et al., 2013), but also to disease pathogenesis (Itoi, 2008; Nagai et al., 2010). We generated ScFv monoclonal antibodies against three different epitopes of Ro60 as recombinant fusion proteins with PE40, which catalyzed the ADP-ribose of nicotinamide adenine dinucleotide transformation into protein translation prolonged factor2, thus preventing protein synthesis and leading to cell death (Armstrong and Merrill, 2004; Yates and Merrill, 2004). The ScFv monoclonal antibodies combined with special epitopes in the LCs, leading to their death as measured by an MTT reduction assay which is a common assay for assessing cell viability (Huang et al., 2012; Zhu et al., 2013).

Various factors have been shown to result in the abnormal expression of Ro antigens on the cell surface, and Reed et al. $(2008,2010)$ held that LCs expressed different Ro60 apotopes. Furthermore, Kurien et al. (2012) found that a cross-antigenicity existed between Ro60 and spectrin. These results revealed that the LC membrane might express different Ro60 epitopes or have cross-antigenicity with other antigens.

In this study, the cell toxicity of ITs to LCs was determined by the degree of expression of relevant Ro60 epitopes in the LC membrane. Results showed that the three ITs promoted significant cell toxicity to the LCs of healthy controls compared to that produced by ALB. We conjectured that Ro antigen and/or other antigens with cross-antigenicity to Ro60 were expressed on the surface of normal or apoptotic LCs. Significant differences in the cytotoxicities of the LCs from patients with SLE and pSS compared to the controls were observed for the three ITs excluding AE1. AE2 and AE3 showed significant toxicity to pSS and LSE LCs, respectively, which was in accordance with the relative decreased LC count seen in each disorder. The results also suggested that two diseases expressed different Ro60 epitopes or had cross-antigenicity with different antigens. Further investigation of autoantibodies to the three epitopes in the two groups demonstrated that there was no difference in the positive rates. We therefore hypothesized that the LC decrease was not always influenced by the different distribution of antibodies in patients with SLE and pSS, but might be due to the difference in differential expression of the LC epitopes. Thus, the results of this study suggested that the LC decrease in patients with SLE and pSS is associated with Ro60 antibodies, but that the mechanisms of LC decrease in these two common autoimmune diseases are quite different.

\section{Conflicts of interest}

The authors declare no conflict of interest. 


\section{ACKNOWLEDGMENTS}

Research supported by the Project of Anhui Provincial Key Clinical Medical Disciplines (\#2008N012) and the Talent Fund of the First Affiliated Hospital of Anhui Medical University (\#AYFY07018).

\section{REFERENCES}

Arbuckle MR, McClain MT, Rubertone MV, Scofield RH, et al. (2003). Development of autoantibodies before the clinical onset of systemic lupus erythematosus. N. Engl. J. Med. 349: 1526-1533.

Armstrong S and Merrill AR (2004). Toward the elucidation of the catalytic mechanism of the mono-ADP-ribosyltransferase activity of Pseudomonas aeruginosa exotoxin A. Biochemistry 43: 183-194.

Hochberg MC (1997). Updating the American College of Rheumatology revised criteria for the classification of systemic lupus erythematosus. Arthritis Rheum. 40: 1725.

Huang J, Li YM, Massague J, Sicheneder A, et al. (2012). Intracerebral infusion of the bispecific targeted toxin DTATEGF in a mouse xenograft model of a human metastatic non-small cell lung cancer. J. Neurooncol. 109: 229-238.

Huang SC, Yu H, Scofield RH and Harley JB (1995). Human anti-Ro autoantibodies bind peptides accessible to the surface of the native Ro autoantigen. Scand. J. Immunol. 41: 220-228.

Itoi K (2008). Ablation of the central noradrenergic neurons for unraveling their roles in stress and anxiety. Ann. N. Y. Acad. Sci. 1129: 47-54.

Kurien BT, Porter A, Dorri Y, Iqbal S, et al. (2011). Degree of modification of Ro60 by the lipid peroxidation by-product 4-hydroxy-2-nonenal may differentially induce Sjögren syndrome or systemic lupus erythematosus in BALB/c mice. Free Radic. Biol. Med. 50: 1222-1233.

Kurien BT, Dorri Y, Bachmann M and Scofield RH (2012). Induction of anti-Ro60/anti-La by immunisation with spectrin and induction of anti-spectrin by immunisation with Ro60 and 4-hydroxy-2-nonenal-modified Ro60 immunisation. Clin. Exp. Rheumatol. 30: 886-893.

Li YJ, Liu L and Zhang FC (2003). The clinical significance of SSA antigen and its different positive expressions. Zhonghua Nei Ke Za Zhi 42: 165-168.

Li HB, Zhang X, Tang FL and Zhang FC (2010). The soluble expression and identification of single-chain fragment V antibodies against SSA antigen epitopes from the pHEN2 phagemid library. Zhonghua Nei Ke Za Zhi 49: 614-617.

Nagai T, Tanaka M, Hasui K, Shirahama H, et al. (2010). Effect of an immunotoxin to folate receptor beta on bleomycininduced experimental pulmonary fibrosis. Clin. Exp. Immunol. 161: 348-356.

Reed JH, Jackson MW and Gordon TP (2008). A B cell apotope of Ro 60 in systemic lupus erythematosus. Arthritis Rheum. 58: 1125-1129.

Reed JH, Dudek NL, Osborne SE, Kaufman KM, et al. (2010). Reactivity with dichotomous determinants of Ro 60 stratifies autoantibody responses in lupus and primary Sjögren's syndrome. Arthritis Rheum. 62: 1448-1456.

Scofield RH, Zhang FC, Kurien BT and Harley JB (1997). Anti-Ro fine specificity defined by multiple antigenic peptides identifies components of tertiary epitopes. Clin. Exp. Immunol. 109: 480-487.

Shuai ZW, Zhang FC, Tang FL and Zhang X (2008). An investigation on the pathogenic mechanisms of autoantibodies against different epitopes on 60KD SSA/Ro antigen in related autoimmune diseases. Int. J. Rheum. Dis. 11: A483 P3O-P03.

Vitali C, Bombardieri S, Jonsson R, Moutsopoulos HM, et al. (2002). Classification criteria for Sjögren's syndrome: a revised version of the European criteria proposed by the American-European Consensus Group. Ann. Rheum. Dis. 61: 554-558.

Yates SP and Merrill AR (2004). Elucidation of eukaryotic elongation factor-2 contact sites within the catalytic domain of Pseudomonas aeruginosa exotoxin A. Biochem. J. 379: 563-572.

Yoshimi R, Ueda A, Ozato K and Ishigatsubo Y (2012). Clinical and pathological roles of Ro/SSA autoantibody system. Clin. Dev. Immunol. 2012: 606195.

Zhou Z, Zhang FC, Tang FL and Dong Z (2003). Construction and identification of anti-SSA antibody phage libraries. Zhonghua Feng Shi Bing Xue Za Zhi 7: 394-398.

Zhu X, Tao K, Li Y, Li S, et al. (2013). A new recombinant immunotoxin hscFv-ETA' demonstrates specific cytotoxicity against chronic myeloid leukemia cells in vitro. Immunol. Lett. 154: 18-24. 